\title{
Impact of magnetic field on shear viscosity of quark matter in Nambu-Jona-Lasinio model
}

\author{
Sabyasachi Ghosh, ${ }^{1}$ Payal Mohanty, ${ }^{2}$ Bhaswar Chatterjee, ${ }^{3}$ Arghya Mukharjee, ${ }^{4}$ and Hiranmaya Mishra ${ }^{5}$ \\ ${ }^{1}$ Indian Institute of Technology Bhilai, GEC Campus, Sejbahar, Raipur 492015, Chhattisgarh, India \\ ${ }^{2}$ National Institute of Science Education and Research, HBNI, 752050 Odisha, India \\ ${ }^{3}$ Department of Physics, Indian Institute of Technology Roorkee, Roorkee 247 667, India \\ ${ }^{4}$ Saha Institute of Nuclear Physics, 1/AF Bidhannagar, Kolkata 700064, India \\ ${ }^{5}$ Theory Division, Physical Research Laboratory, Navrangpura, Ahmedabad 380 009, India
}

(Received 10 July 2018; revised manuscript received 11 January 2019; published 27 August 2019)

\begin{abstract}
We have investigated the shear viscosity of quark matter in the presence of a strong uniform magnetic field background in which the Nambu-Jona-Lasinio model has been considered to describe the magnetothermodynamical properties of the medium. In the presence of magnetic field, the shear viscosity coefficient gets split into different components because of anisotropy in tangential stress of the fluid. Four different components can be merged to two components in the strong field limit, at which the collisional width of the quark becomes much lower than its synchrotron frequency. A simplified contact diagram of quark-quark interaction can estimate a small collisional width, for which the strong field limit expressions are exactly applicable, although for the Relativistic Heavy Ion Collider or LHC matter, one can expect a large thermal width, for which generalized four-component viscosities are necessary. We have explored all these different possible cases in the thermodynamical framework of the Nambu-Jona-Lasinio model.
\end{abstract}

DOI: $10.1103 /$ PhysRevD.100.034024

\section{INTRODUCTION}

One of the major updates in the research of heavy-ion collision (HIC) experiments like the Relativistic Heavy Ion Collider (RHIC) and LHC is that the produced medium behaves like a nearly perfect fluid [1], with the smallest shear viscosity-to-entropy density ratio $(\eta / s)$ ever observed in nature. On the other hand, recent progress in the HIC research has speculated that the produced medium may face a high magnetic field [2] in the noncentral heavy-ion collisions. The possible space-time dependence of this produced magnetic field has been investigated in Refs. [3-7]. A considerable amount of research work has already been performed in understanding the influence of the magnetic field on the QCD phase diagram. See, for example, the review article [8] for recent updates. The modification of the QCD phase diagram in the presence of magnetic field is directly related to the corresponding change in the quark condensate, and its enhancement with magnetic field is known as magnetic catalysis, which is a quite expected feature in vacuum as well as at finite temperature [9-14]. However, recent

Published by the American Physical Society under the terms of the Creative Commons Attribution 4.0 International license. Further distribution of this work must maintain attribution to the author(s) and the published article's title, journal citation, and DOI. Funded by SCOAP ${ }^{3}$. calculations, based on lattice QCD [15,16], have found inverse magnetic catalysis, the possibility of which is also indicated by some effective QCD model calculations [17-20]. The modifications pertaining to the QCD phase diagram may also have some impact in the transport properties of the medium produced in HICs. In the presence of magnetic field, different transport coefficients like shear viscosity [21-28], bulk viscosity [26-30], and electrical conductivity [31-38] of quark matter are calculated in recent times. The simulation of magnetohydrodynamics $[39,40]$ as well as the transport simulation for an external magnetic field [41] may require these temperature- and magnetic field-dependent transport coefficients for their future upgrading.

Among the different transport coefficients, only the shear viscosity is our matter of interest in the present work, in which a two-flavor Nambu-Jona-Lasinio (NJL) model has been used as a dynamical framework. Among the earlier calculations of shear viscosity for magnetized matter [21-28,42], we find that Refs. [21-24] have not explored its component decomposition, which is explicitly analyzed in Refs. [25-28,42]. This component decomposition of shear viscosity due to anisotropy, created by external magnetic field or other sources, is well studied in the direction of gauge gravity duality (see Refs. [43,44] and references therein).

We have first followed the strong field limit expression, obtained in Refs. [25,45], in which four components of 
shear viscosity merge to two main components, as also found in gauge gravity dual theory [43,44]. One is normaltype shear viscosity, and another is a Hall-type component. The normal component depends on both collisional and synchrotron frequencies, but the Hall component depends completely on the synchrotron frequency in the strong field limit. However, below that strong field limit, both components can depend on both frequencies. We have also studied the general structure of four different components in the moderate field zone, which is expected in RHIC or LHC experiments.

The article is organized as follows. In Sec. II, the background formalism of the NJL model is addressed. Next, Sec. III covers the formalism of shear viscosity for the case of strong magnetic field in Sec. III A and then its corresponding numerical outcome in Sec. III B. Realizing the strong field limit cannot be applicable for RHIC or LHC matter, which should have small collisional relaxation time, we go through the strong field case to general case, the modified formalism and corresponding numerical outcome of which are discussed in Secs. IV A and IV B, respectively. At the end, investigations of all these different possible cases are summarized in Sec. V.

\section{NJL MODEL IN THE PRESENCE OF MAGNETIC FIELD}

We shall consider here the two-flavor ( $u$ and $d$ quarks) NJL model with a determinant interaction with the Lagrangian density given as $[12,46]$

$$
\begin{aligned}
\mathcal{L}= & \bar{\psi}(i \not D-m) \psi+G \sum_{a=0}^{3}\left[\left(\bar{\psi} \tau^{a} \psi\right)^{2}+\left(\bar{\psi} i \gamma_{5} \tau^{a} \psi\right)^{2}\right] \\
& +K\left[\operatorname{det}_{f} \bar{\psi}\left(1+\gamma_{5}\right) \psi+\operatorname{det}_{f} \bar{\psi}\left(1-\gamma_{5}\right) \psi\right]
\end{aligned}
$$

where $\psi=(u, d)^{T}$ is the doublet of quarks and $m=$ $\left(m_{u}, m_{d}\right)$ is the current quark mass with $m_{u}=m_{d}$. The first term is basically the Dirac Lagrangian in the presence of an external magnetic field, which we assume to be constant and in the direction of the $z$ axis. For calculational purposes, we shall further choose the gauge such that the corresponding electromagnetic potential is given by $A_{\mu}(\boldsymbol{x})=(0,0, B x, 0)$. The second line is the attractive part of the quark-antiquark channel of the Fiertz-transformed color current-current interaction. The third line is the 't Hooft determinant interaction in the flavor space that describes the effects of instantons and is flavor mixing. $\tau^{a}$, $a=0 \cdots 3$ are the $\mathrm{U}(2)$ generators in the flavor space. In the absence of magnetic field, the interaction is invariant under $S U(2)_{L} \times S U(2)_{R} \times U_{V}(1)$. The second term has an additional $U(1)_{A}$ symmetry, while the 't Hooft term does not have this symmetry and reflects the $U(1)_{A}$ anomaly of QCD.

The thermodynamic potential corresponding to Eq. (1) can be computed exactly in the same manner as was done previously in Ref. [13], which was done for three flavors in a variational method with an explicit structure for the vacuum with quark-antiquark condensates. The thermodynamic potential is then given as

$$
\begin{aligned}
\Omega= & \sum_{i} \Omega_{0}^{i}+\sum_{i} \Omega_{\text {field }}^{i}+\sum_{i} \Omega_{\text {med }}^{i} \\
& +2 G \sum_{i} I_{s}^{i 2}+2 K I_{s}^{u} I_{s}^{d},
\end{aligned}
$$

where $i$ is the flavor index. We might mention here that the above thermodynamic potential can also be derived in a mean field approximation [46]. The vacuum term for $i$ th flavor $\Omega_{0}^{i}$ is given as

$$
\begin{aligned}
\Omega_{0}^{i}= & -\frac{2 N_{c}}{(2 \pi)^{3}} \int d \mathbf{p} \sqrt{\mathbf{p}^{2}+M_{i}^{2}} \theta(\Lambda-|\mathbf{p}|) \\
= & -\frac{N_{c}}{8 \pi^{2}}\left[\Lambda \sqrt{\Lambda^{2}+M_{i}^{2}}\left(2 \Lambda^{2}+M_{i}^{2}\right)\right. \\
& \left.-M_{i}^{4} \log \frac{\Lambda+\sqrt{\Lambda^{2}+M_{i}^{2}}}{M_{i}}\right],
\end{aligned}
$$

with $\Lambda$ as the three-momentum cutoff associated with the NJL model. The field contribution that arises from the effect of magnetic field on the Dirac vacuum is given by

$\Omega_{\text {field }}^{i}=-\frac{N_{c}}{2 \pi^{2}} \sum_{i}\left|q_{i} B\right|^{2}\left[\zeta^{\prime}\left(-1, x_{i}\right)-\frac{1}{2}\left(x_{i}^{2}-x_{i}\right) \ln x_{i}+\frac{x_{i}^{2}}{4}\right]$,

where we have defined a dimensionless quantity, $x_{i}=$ $M_{i}^{2} / 2\left|q_{i} B\right|$, i.e., the mass parameter in units of magnetic field, and $\zeta^{\prime}(-1, x)=d \zeta(z, x) /\left.d z\right|_{z=1}$ is the derivative of the Riemann-Hurwitz $\zeta$ function, which is given by

$$
\begin{aligned}
\zeta^{\prime}(-1, x)= & \frac{\ln x}{2}\left[x^{2}-x+\frac{1}{6}\right]-\frac{x^{2}}{4} \\
& +x^{2} \int_{0}^{\infty} \frac{2 \tan ^{-1} y+y \ln \left(1+y^{2}\right)}{e^{2 \pi x y}-1} d y .
\end{aligned}
$$

Finally, the medium contribution $\Omega_{\text {med }}^{i}$ is given as

$$
\Omega_{\text {med }}^{i}=\frac{-N_{c}}{\pi^{2}} \sum_{n=0}^{n_{\max }} \frac{\alpha_{n}\left|q_{i} B\right|}{\beta} \int d p_{z} \log \left(1+e^{-\beta \omega_{n}^{i}}\right)
$$

with the single particle energy in the presence of magnetic field $\omega_{n}^{i}=\sqrt{p_{z}^{2}+2 n\left|q_{i}\right| B+m^{2}}$. The condition of a sharp three-momentum cutoff translates to a finite number of Landau-level summation with $n_{\max }=\operatorname{Int}\left[\frac{\Lambda^{2}}{2\left|q_{i}\right| B}\right]$ when $p_{z}=0$. Further, for the medium contributions, this also leads to a cutoff for the $\left|p_{z}\right|$ as $\Lambda^{\prime}=\sqrt{\Lambda^{2}-2 n\left|q_{i}\right| B}$ for a given value of $n$. 
Similarly, in Eq. (2), the quark condensate $I_{s}^{i}=-\left\langle\bar{\psi}^{i} \psi^{i}\right\rangle$ can be separated into a zero field vacuum term, a finite field-dependent term, and a medium dependent term as

$$
\begin{aligned}
I_{s}^{i} \equiv & -\left\langle\bar{\psi}^{i} \psi^{i}\right\rangle=\frac{2 N_{c}}{(2 \pi)^{3}} \int_{|\mathbf{p}|<\Lambda} d \boldsymbol{p} \frac{M^{i}}{\sqrt{\boldsymbol{p}^{2}+M^{i 2}}} \\
& +\frac{N_{c} M^{i}\left|q^{i} B\right|}{(2 \pi)^{2}}\left[x^{i}\left(1-\ln x^{i}\right)+\ln \Gamma\left(x^{i}\right)+\frac{1}{2} \ln \frac{x^{i}}{2 \pi}\right] \\
& -\sum_{n=0}^{n_{\max }} \frac{N_{c}\left|q^{i}\right| B \alpha_{n}}{(2 \pi)^{2}} \int d p_{z} \frac{M^{i}}{\omega_{i}^{n}} \frac{1}{1+\epsilon^{-\beta \omega_{i}^{n}}} \\
= & I_{\text {svac }}^{i}+I_{\text {sfield }}^{i}+I_{\text {smed }}^{i} .
\end{aligned}
$$

The zero field vacuum contribution, $I_{s \text { vac }}^{i}$, can be analytically calculated using a sharp momentum cutoff $\Lambda$ and can be written as

$$
I_{S_{\mathrm{vac}}}^{i}=\frac{N_{c} M_{s}^{i}}{2 \pi^{2}}\left[\Lambda \sqrt{\Lambda^{2}+M^{i 2}}-M^{i 2} \log \left\{\frac{\Lambda+\sqrt{\Lambda^{2}+M^{i 2}}}{M^{i}}\right\}\right] .
$$

The constituent quark mass $M^{i}$ satisfies the gap equation

$$
M_{i}=m_{i}+4 G I_{s}^{i}+2 K\left|\epsilon^{i j}\right| I_{s}^{j} .
$$

This completes the definitions of all the quantities that are used to describe the thermodynamic potential in Eq. (2).

For numerical evaluations, we choose the parameters as in Ref. [46]; i.e., we write $G=(1-\alpha) G_{0}$ and $K / 2=\alpha G_{0}$. The parameter $\alpha$ controls the strength of the instanton interaction, while the value of the quark condensate is determined by the combination of parameters: $m=6 \mathrm{MeV}$, the three-momentum cut off $\Lambda=590 \mathrm{MeV}$, and the dimensionless coupling $G_{0} \Lambda^{2}=2.435$. These values lead to pion mass in vacuum as $140.2 \mathrm{MeV}$, a pion decay constant of $92.6 \mathrm{MeV}$, and quark condensate $\langle\bar{u} u\rangle=\langle\bar{d} d\rangle=(-241.5 \mathrm{MeV})^{3}$, all in reasonable agreement with the experimental values. This also leads to a vacuum constituent quark mass of $400 \mathrm{MeV}$. Further, in all these calculations, we have taken $\alpha=0.15$ as a reasonable value interpolated from $\eta-\eta^{\prime}$ splitting within the threeflavor NJL model [46].

Figure 1(a) shows the constituent quark mass as a function of temperature for different values of magnetic fields. At $e B=0$, masses of $u$ and $d$ quarks exactly coincide (dotted line), while for nonzero $e B$, they split due to different electrical charges of the two quark flavors, and their splitting increases with the magnetic field. Our results reveal the magnetic catalysis in the entire temperature range, and therefore the transition temperature $T_{c}$ increases with $B$. Using this $M_{Q}(T, e B)$, one can calculate the entropy density $s$ with the help of a quasiparticle relation,

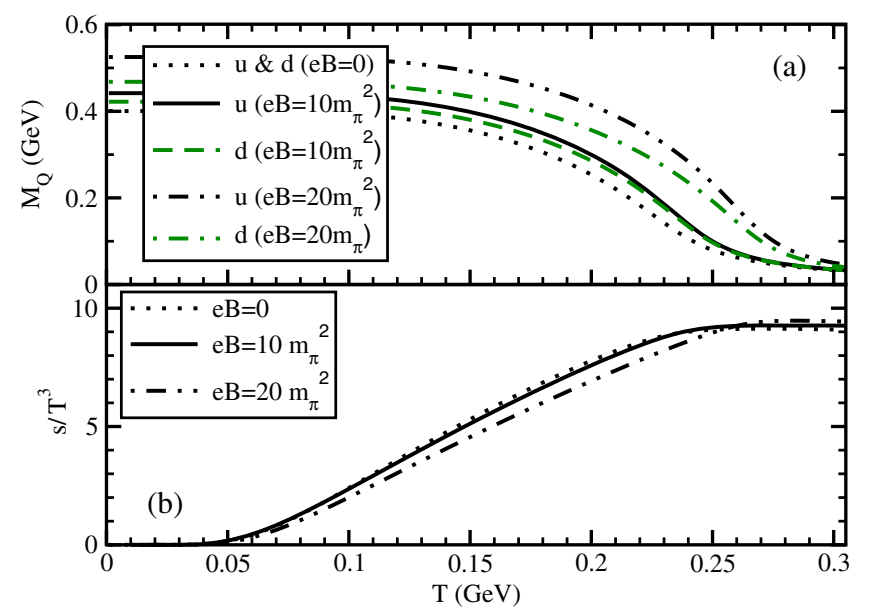

FIG. 1. $T$ dependence of constituent quark masses $\left(M_{Q}\right)$ and normalized entropy density $\left(s / T^{3}\right)$ for different values of magnetic fields.

$s=\frac{N_{c}}{\pi^{2} T} \sum_{i=u, d} \sum_{n=0}^{n_{\max }} \alpha_{n}\left|q_{i}\right| B \int d \boldsymbol{k}_{z}\left[\frac{\boldsymbol{k}_{z}^{2}}{\omega_{n}^{i}}+\omega_{n}^{i}\right] f_{0}\left(\omega_{n}^{i}\right)$,

where $f_{0}\left(\omega_{n}^{i}\right)$ is the Fermi-Dirac distribution function. The temperature dependence of normalized entropy density $s / T^{3}$ for $e B=0$ (dotted line), $10 m_{\pi}^{2}$ (solid line), and $20 m_{\pi}^{2}$ (dash-dotted line) is shown in Fig. 1(b). We notice that $s$ decreases as $e B$ increases in the lower-temperature domain, but all the curves are merged into its StefanBoltzmann limit in the high-temperature region.

\section{STRONG MAGNETIC FIELD CASE}

\section{A. Formalism of shear viscosity in the presence of strong magnetic field}

Let us first briefly recapitulate the relaxation time approximation technique to calculate shear viscosity coefficients of a relativistic fluid in the absence of any magnetic field (i.e., $B=0$ ), which is elaborately given in Refs. [47,48]. Then, we will come to its corresponding formalism in presence of the strong magnetic field, well described in Refs. [25,45].

The total energy-momentum tensor of relativistic fluid, $T^{\mu \nu}=T_{0}^{\mu \nu}+T_{D}^{\mu \nu}$, contains the ideal part $T_{0}^{\mu \nu}=$ $-P g^{\mu \nu}+(P+\epsilon) u^{\mu} u^{\nu}$ and dissipation part $T_{D}^{\mu \nu}=\eta U^{\mu \nu}$ (only shear dissipation), where $P, \epsilon$, and $u^{\mu}$ are, respectively, the pressure, energy density, and 4-velocity of the fluid. The tensor structure $U^{\mu \nu}$, linked with shear viscosity $\eta$, has the form [48]

$$
\begin{aligned}
U^{\mu \nu} & =D^{\mu} u^{\nu}+D^{\nu} u^{\mu}+\frac{2}{3} \Delta^{\mu \nu} \partial_{\sigma} u^{\sigma} \quad \text { with } \\
D^{\mu} & =\partial^{\mu}-u^{\mu} u^{\sigma} \partial_{\sigma}, \quad \Delta^{\mu \nu}=u^{\mu} u^{\nu}-g^{\mu \nu} .
\end{aligned}
$$

Now, in terms of four-momentum $k^{\mu}=(\omega, \boldsymbol{k})$ and thermal distribution function $f_{0}=1 /\left\{e^{\beta \omega}+1\right\}$ of a quark 
at temperature $T=1 / \beta$, one can express the total energymomentum tensor as

$$
T^{\mu \nu}=\int \frac{d^{3} \boldsymbol{k}}{(2 \pi)^{3}} \frac{k^{\mu} k^{\nu}}{\omega}\left\{f_{0}+\phi f_{0}\left(1-f_{0}\right)\right\},
$$

where the second term in the curly brackets involving the function $\phi$ describes the nonequilibrium part for which one can construct the shear dissipative part $T_{D}^{\mu \nu}$ of the energymomentum tensor [48]. In terms of velocity gradient tensor $U^{\mu \nu}$, the function $\phi$ can be written as $\phi=C k_{\mu} k_{\nu} U^{\mu \nu}$. The unknown $C$ can be obtained as $C=\frac{\tau_{c} \beta}{2 \omega}$ by using the relativistic Boltzmann equation (RBE), where $\tau_{c}$ is the relaxation time of the quark in the medium. Comparing the coefficients of $U^{\mu \nu}$ from the dissipative part of the energymomentum tensor, we finally obtain the expression of the shear viscosity coefficient as

$$
\eta=\frac{g \beta}{15} \int \frac{d^{3} \boldsymbol{k}}{(2 \pi)^{3}} \frac{\boldsymbol{k}^{4}}{\omega^{2}} \tau_{c} f_{0}\left(1-f_{0}\right),
$$

where $g=2 \times 2 \times 2 \times 3$ is an additional input that takes care of the degeneracy factor for two-flavor (isospin symmetric) quark matter.

Now, let us discuss the effect of external magnetic field on the shear viscosity of the medium. In the presence of a constant background magnetic field, the medium can possess five independent components of shear viscosity, and the dissipative part of the energy-momentum tensor (in 3 -vector notation) can be written as $[25,45]$

$$
T_{D}^{i j}=\sum_{n=0}^{4} \eta_{n} V_{n}^{i j}=\int \frac{d^{3} \boldsymbol{k}}{(2 \pi)^{3}} \frac{k^{i} k^{j}}{\omega} \delta f,
$$

where

$$
\delta f=\phi f_{0}\left(1-f_{0}\right)=\sum_{n=0}^{4} C_{n} k^{i} k^{j} V_{n}^{i j} f_{0}\left(1-f_{0}\right)
$$

and

$$
\phi=\sum_{n=0}^{4} C_{n} k^{i} k^{j} V_{n}^{i j}
$$

is assumed in terms of same tensorial components $V_{n}^{i j}$.

Among these five components, four components $(n=1, \ldots, 4)$ will be only our matter of interest as they only depend on magnetic field, while the $n=0$ component remains undisturbed by magnetic field. This $n=0$ viscosity component can be compared with electrical/ thermal conductivity along the direction of magnetic field, as discussed Refs. [34,45], in which they also remain undisturbed by the external magnetic field. Hence, ignoring the $\eta_{0}$ or $V_{0}^{i j}$ component [25,45], one can obtain four shear viscosity coefficients as

$\eta_{(n=1,2,3,4)}^{i}=\frac{2 g_{i}}{15} \int \frac{d^{3} \boldsymbol{k}}{(2 \pi)^{3}} \frac{\boldsymbol{k}^{4}}{\omega^{i}} C_{(n=1,2,3,4)}^{i} f_{0}^{i}\left(1-f_{0}^{i}\right)$,

where the unknown $C_{n}^{i}$ again will be determined with the help of the RBE but in a two-step approximations. Since the magnetic field will destroy the degeneracy of $u$ and $d$ quark masses, the energy $\omega^{i}$, distribution function $f_{0}^{i}$, and $C_{n}^{i}$ in Eq. (17) carry the flavor index $i$. The $g_{i}=2 \times 2 \times 3$ is the degeneracy factor of each flavor.

As a first approximation, the particle relaxation time $\tau_{c}$ in the RBE is ignored by assuming that the deviation from equilibrium due to the strong magnetic field is much larger than that due to the particle collisions. Therefore, we get a magnetic field-induced relaxation time $\tau_{B}^{i}=1 / \omega_{B}^{i}$, where

$\omega_{B}^{i}=q_{i} B / \omega^{i}, \quad\left(q_{i}=+\frac{2}{3} e,-\frac{1}{3} e\right.$ for $\left.i=u, d\right)$

is the synchrotron frequency of the quark. So, the strong field limit will be established if we can show that $\tau_{c}^{i} \gg \tau_{B}^{i}$. As a first approximation of RBE [25,45], we ignore $\tau_{c}^{i}$ leading to the the coefficients $C^{i}$ getting related to the field induced relaxation time $\tau_{B}^{i}$ as

$$
C_{1}^{i}=C_{2}^{i}=0, \quad \text { and } \quad C_{4}^{i}=2 C_{3}^{i}=\frac{\tau_{B}^{i} \beta}{2 \omega^{i}}
$$

Next, in the second approximation, a collisional or thermal width $\Gamma_{c}^{i}=1 / \tau_{c}^{i}$, obeying the inequality $\Gamma_{c}^{i} \ll \omega_{B}^{i}$ or $\tau_{c}^{i} \gg \tau_{B}^{i}$, is considered, which leads to the relation [25]

$$
C_{2}^{i}=4 C_{1}^{i}=\frac{\Gamma_{c}^{i}}{\omega_{B}^{i}} C_{4}^{i}=\frac{\Gamma_{c}^{i}}{2 \omega_{B}^{i}} C_{3}^{i},
$$

with $C_{4}^{i}=2 C_{3}^{i}=\frac{\tau_{B}^{i} \beta}{2 \omega^{i}}$. Thus, in the presence of constant background magnetic field $B$, the expressions of the four components of the shear viscosity for the $i=u / d$ quark are

$$
\begin{aligned}
\eta_{2}^{i} & =4 \eta_{1}^{i} \\
& =\frac{g_{i} \beta}{15} \int \frac{d^{3} \boldsymbol{k}}{(2 \pi)^{3}}\left[f_{0}^{i}\left\{1-f_{0}^{i}\right\}\right]\left\{\left(\frac{\Gamma_{c}^{i}}{\omega_{B}^{i}}\right)\left(\frac{1}{\omega_{B}^{i}}\right)\right\}\left(\frac{\boldsymbol{k}^{2}}{\omega^{i}}\right)^{2},
\end{aligned}
$$

and 


$$
\begin{aligned}
\eta_{4}^{i} & =2 \eta_{3}^{i} \\
& =\frac{g_{i} \beta}{15} \int \frac{d^{3} \boldsymbol{k}}{(2 \pi)^{3}}\left[f_{0}^{i}\left\{1-f_{0}^{i}\right\}\right]\left\{\frac{1}{\omega_{B}^{i}}\right\}\left(\frac{\boldsymbol{k}^{2}}{\omega^{i}}\right)^{2} .
\end{aligned}
$$

If we compare Eqs. (21) and (22) with Eq. (13), then we can get a physical interpretation of these shear viscosity components. In the plane perpendicular to the external magnetic field, the momentum transfer due to shear stress is independent of the particle collisions and will be proportional to the field-induced relaxation $\left(\tau_{B}=1 / \omega_{B}\right)$, which is basically the inverse of the synchrotron frequency. In other words, rotational motion of the charged particles with corresponding synchrotron frequency provides the required momentum transfer for generating shear stress along the tangential directions, located in the perpendicular plane with respect to the magnetic field. This strength of the shear stress, velocity gradient, and its proportional coefficients $\eta_{3}$ and $\eta_{4}$ are completely originated due to (strong) magnetic field background.

In other possible tangential directions, both the collisional and rotational energies take part in momentum transfer. Therefore, the fraction $\Gamma_{c} / \omega_{B}$ is required for fixing the proportional strength of viscosities $\eta_{1}$ and $\eta_{2}$. The corresponding relaxation time for these components becomes $\left[\left(\frac{\Gamma_{c}}{\omega_{B}}\right) \frac{1}{\omega_{B}}\right]^{-1}$.

\section{B. Results of strong field case}

In the strong field limit, the $\tau_{c}$ should be much, much greater than $\tau_{B}=1 / \omega_{B}=\omega_{Q}^{k} / e_{Q} B=\left\{\boldsymbol{k}^{2}+m_{Q}^{2}\right\}^{1 / 2} / e_{Q} B$ [25], which is basically the inverse of the synchrotron frequency $\omega_{B}$. The assumption $\tau_{c} \gg \tau_{B}$ is the baseline of the strong field case formalism, discussed earlier in Sec. III A. After calculating $\tau_{c}$ microscopically, we can know whether or not the value of $\tau_{c}$ satisfies $\tau_{c} \gg \tau_{B}$ or the strong field limit. Here, we will attempt to obtain $\tau_{c}(T, e B)$ in an explicit microscopic calculation. For this purpose, let us start with the $B=0$ case with the standard expression of collisional relaxation time $\tau_{c}$ or thermal width,

$$
\begin{aligned}
\Gamma_{c}\left(T, \boldsymbol{k}_{a}\right)= & \frac{1}{\tau_{c}} \\
= & \sum_{b} \int \frac{d^{3} k_{b}}{(2 \pi)^{3}} \sigma_{a b}\left(T, \boldsymbol{k}_{a}, \boldsymbol{k}_{b}\right) v_{a b}\left(T, \boldsymbol{k}_{a}, \boldsymbol{k}_{b}\right) \\
& \times f_{b}\left(T, \boldsymbol{k}_{b}\right),
\end{aligned}
$$

where

$v_{a b}\left(T, \boldsymbol{k}_{a}, \boldsymbol{k}_{b}\right)=\frac{\left\{\left(\omega_{a}+\omega_{b}\right)^{2}-4 M_{Q}^{2}(T)\right\}^{1 / 2}\left(\omega_{a}+\omega_{b}\right)}{2 \omega_{a} \omega_{b}}$ is the relative velocity with $\omega_{a, b}=\left\{\boldsymbol{k}_{a, b}^{2}+M_{Q}^{2}(T)\right\}^{1 / 2}$. To map grossly the scattering strength of NJL dynamics, let us calculate cross section $\sigma_{a b}$ from a simple four-quark contact diagram, shown inside Fig. 2(b). To do this, we use the standard quantum field theoretical relation of $2 \rightarrow 2$ scattering,

$$
\sigma_{a b}=\frac{1}{16 \pi s} \overline{|M|_{a b}^{2}}
$$

where $s=\left(\omega_{a}+\omega_{b}\right)^{2}$ and

$\overline{|M|_{a b}^{2}}=\frac{1}{2 \times 2} G^{2} 16\left(\frac{s}{2}\right)^{2}=G^{2} s^{2}, \quad s=\left(\omega_{a}+\omega_{b}\right)^{2}$.

Hence, we get a temperature- and momentum-dependent cross section $\sigma_{a b}\left(T, \boldsymbol{k}_{a}, \boldsymbol{k}_{b}\right)=\frac{G^{2}}{16 \pi} s\left(T, \boldsymbol{k}_{a}, \boldsymbol{k}_{b}\right)$.

By maintaining electric charge conservation, we will get 12 possible $2 \rightarrow 2\left(a b \rightarrow a^{\prime} b^{\prime}\right)$ scattering processes:

$u \bar{u} \rightarrow u \bar{u}, \quad u \bar{d} \rightarrow u \bar{d}, \quad u \bar{u} \rightarrow d \bar{d}, \quad u u \rightarrow u u$,

$u d \rightarrow u d, \quad \bar{u} \bar{u} \rightarrow \bar{u} \bar{u}, \quad \bar{u} \bar{d} \rightarrow \bar{u} \bar{d}, \quad d \bar{d} \rightarrow d \bar{d}$,

$d \bar{d} \rightarrow u \bar{u}, \quad d \bar{u} \rightarrow d \bar{u}, \quad d d \rightarrow d d, \quad \bar{d} \bar{d} \rightarrow \bar{d} \bar{d}$.

So, fixing any initial particle $a$ as the probe particle, we have to take the summation of $b$ to calculate $\Gamma_{c}\left(T, \boldsymbol{k}_{a}\right)$ as in Eq. (23) Taking the momentum average of the probe particle, we get only the $T$-dependent quark width,

$$
\Gamma_{c}(T)=\frac{1}{\tau_{c}}=\frac{\int \frac{d^{3} k_{a}}{(2 \pi)^{3}} \Gamma\left(T, \boldsymbol{k}_{a}\right) f_{a}\left(T, \boldsymbol{k}_{a}\right)}{\int \frac{d^{3} k_{a}}{(2 \pi)^{3}} f_{a}\left(T, \boldsymbol{k}_{a}\right)} .
$$

So, we find that temperature dependence mainly comes from thermodynamical phase space and $M_{Q}(T)$. If we go

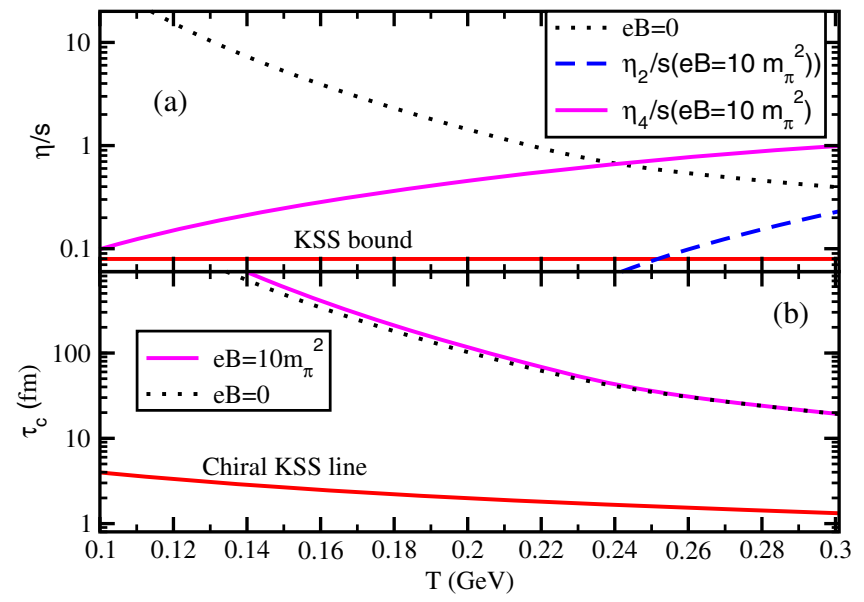

FIG. 2. (a) $T$ dependence of $\eta / s$ at $e B=0$ (dotted line) and $\eta_{2} / s$ (blue dash line) and $\eta_{4} / s$ (pink solid line) at $e B=10 m_{\pi}^{2}$. (b) $T$ dependence of $\tau_{c}$ at $e B=0$ (dotted line) and $e B=10 m_{\pi}^{2}$ (pink solid line). 
for a simplified extension of the finite magnetic field picture by replacing $M_{Q}(T, e B)$ in Eq. (28), we can get $\Gamma_{c}(T, e B)$. Figure 2(b) shows the $\tau_{c}(T, e B)$ at $e B=0$ (black dotted line) and $e B=10 m_{\pi}^{2}$ (pink solid line). Due to increase in the number density of the particles with temperature, collisional frequency increases, and relaxation time decreases with $T$. The $e B$ dependence of $\tau_{c}$ enters via $e B$ dependence of constituent quark mass $M_{Q}(e B)$. Increasing function $M_{Q}(e B)$ can suppress the number density, which makes $\Gamma_{c}$ decrease and $\tau_{c}$ increase with $e B$. Being proportional with the decreasing function $\tau_{c}(T)$ for $e B=0, \eta / s$ decreases with $T$. Let us note that in the behavior of $\eta / s$ with temperature arises from two competing quantities that depend upon temperature. Due to thermodynamic phase space factor, this ratio is an increasing function of temperature while the relaxation time decrease with temperature. For constant relaxation time, due to thermal phase space factor, $\eta / s$ increase with temperature. This can be easily found out for mass less ideal gas behavior of the expression for $\eta / s$. On the otherhand, for the decreasing behavior of temperature dependent $\tau$, dominates over the increasing behaviour arising from the thermal phase space making the ratio decreasing with temperature, as may be noticed in the dotted line of Fig. 2(a).

Another noticeable thing is that for the contact diagram of $2 \rightarrow 2$ scattering processes, the NJL model estimates very large $\tau_{c}$, which is quite far from the chiral KovtunSon-Starinets (KSS) line $\tau_{c}(T)=\frac{5}{4 \pi T}$, shown by the red solid line in Fig. 2(b). The chiral KSS line comes from the requirement of $\frac{\eta}{s}=\frac{1}{4 \pi}$ for the massless particles. Therefore, in Fig. 2(a), black dotted line is also quite far from the red horizontal line, denoted KSS value for the ratio $\frac{\eta}{s}=\frac{1}{4 \pi}$.

For this high value of $\tau_{c}, e B=10 m_{\pi}^{2}$ can be considered as strong field limit case because $\tau_{B}$ remains within the range $0.8-3 \mathrm{fm}$. So, we can safely say that at $e B=10 m_{\pi}^{2}$ we can consider $\tau_{c} \gg \tau_{B}$ or the strong field limit case [25]. It is interesting to notice in Eq. (21) that the position of $\tau_{c}$ for the strong field case becomes inverse $\left(\eta_{2} \propto 1 / \tau_{c}\right)$; therefore, $\eta_{2} / s$ becomes an increasing function of $T$, as shown by the pink solid line in Fig. 2(a). Hence, in the strong field limit, $\eta_{1,2} \propto 1 / \tau_{c}$ follows the opposite trend with respect to the field-free case $\eta \propto \tau_{c}$. When we come to the Hall-type viscosity, $\eta_{3,4} \propto \tau_{B}$, which appears as dissipation free completely as it becomes independent of $\tau_{c} . \eta_{3,4}$ increases with $T$ because of its phase space part, which can be realized from the $\eta_{4} / s$ curve (blue dash line) in Fig. 2(a).

Now, for the simplest contact diagram calculation, we are getting a very large value of $\tau_{c}$, but it cannot be expected in the RHIC or LHC matter, the lifetime of which is approximately $10 \mathrm{fm}$. So, the strong field case cannot be applicable for the RHIC or LHC matter, the $\tau_{c}$ of which is expected to be small, at least smaller than $10 \mathrm{fm}$. We might find alternative possible diagrams, which can provide small $\tau_{c}$. References [49-53] have obtained very small $\tau_{c}$, relevant to the RHIC or LHC matter through a meson exchange-type diagram, the calculation of which in the presence of magnetic field is not at all very straightforward. It might be considered as a future challenging topic. Instead of calculating smaller $\tau_{c}(T)$, we can take it as a parameter and examine the impact of its smaller value. When we consider a small value of $\tau_{c}$ $(<10 \mathrm{fm})$ at $e B=10 m_{\pi}^{2}$, the inequality $\tau_{c} \gg \tau_{B}$ does not hold. So, instead of considering the strong field limit, we might have to find some general structure of $\eta_{n}$, which is attempted in the next section.

\section{FROM STRONG FIELDS TO MODERATE FIELDS}

\section{A. Modified formalism of shear viscosity}

In this section, we will attempt toguess a general structure of shear viscosities, which can be applicable for any value of $\tau_{B}$ and $\tau_{c}$.

We have found that the $\tau_{c}$ in Eq. (13) for $B=0$ is basically replaced by effective relaxation time $\tau_{1,2}^{\text {eff }}=\frac{\tau_{B}^{2}}{\tau_{c}}$ for $\eta_{1,2}$ and $\tau_{3,4}^{\text {eff }}=\tau_{B}$ for $\eta_{3,4}$. Let us take an ansatz of effective relaxations [42],

$$
\begin{array}{ll}
\tau_{1}^{\mathrm{eff}}=\tau_{c} \frac{1}{4\left\{\frac{1}{4}+\left(\tau_{c} / \tau_{B}\right)^{2}\right\}} & \tau_{2}^{\mathrm{eff}}=\tau_{c} \frac{1}{\left\{1+\left(\tau_{c} / \tau_{B}\right)^{2}\right\}} \\
\tau_{3}^{\mathrm{eff}}=\tau_{c} \frac{\tau_{c} / \tau_{B}}{2\left\{\frac{1}{4}+\left(\tau_{c} / \tau_{B}\right)^{2}\right\}} & \tau_{4}^{\mathrm{eff}}=\tau_{c} \frac{\tau_{c} / \tau_{B}}{\left\{1+\left(\tau_{c} / \tau_{B}\right)^{2}\right\}},
\end{array}
$$

which might be considered as their general structure, because in the limit of $\tau_{c} \gg \tau_{B}$, we get

$$
4 \tau_{1}^{\mathrm{eff}}=\tau_{2}^{\mathrm{eff}}=\frac{\tau_{B}^{2}}{\tau_{c}} \quad 2 \tau_{3}^{\mathrm{eff}}=\tau_{4}^{\mathrm{eff}}=\tau_{B} .
$$

It means that we will get back Eqs. (21) and (22) for the strong field limit $\left(\tau_{c} \gg \tau_{B}\right)$.

Using that general structure of relaxation (29) in Eqs. (21) and (22), we will get general expressions of shear viscosity components:

$$
\begin{aligned}
& \eta_{1}=\frac{g \beta}{15} \int \frac{d^{3} \boldsymbol{k}}{(2 \pi)^{3}}\left(\frac{\boldsymbol{k}^{2}}{\omega}\right)^{2} \tau_{c} \frac{1}{4\left\{\frac{1}{4}+\left(\tau_{c} / \tau_{B}\right)^{2}\right\}}\left[f_{0}\left\{1-f_{0}\right\}\right] \\
& \eta_{2}=\frac{g \beta}{15} \int \frac{d^{3} \boldsymbol{k}}{(2 \pi)^{3}}\left(\frac{\boldsymbol{k}^{2}}{\omega}\right)^{2} \tau_{c} \frac{1}{1+\left(\tau_{c} / \tau_{B}\right)^{2}}\left[f_{0}\left\{1-f_{0}\right\}\right] \\
& \eta_{3}=\frac{g \beta}{15} \int \frac{d^{3} \boldsymbol{k}}{(2 \pi)^{3}}\left(\frac{\boldsymbol{k}^{2}}{\omega}\right)^{2} \tau_{c} \frac{\tau_{c} / \tau_{B}}{2\left\{\frac{1}{4}+\left(\tau_{c} / \tau_{B}\right)^{2}\right\}}\left[f_{0}\left\{1-f_{0}\right\}\right] \\
& \eta_{4}=\frac{g \beta}{15} \int \frac{d^{3} \boldsymbol{k}}{(2 \pi)^{3}}\left(\frac{\boldsymbol{k}^{2}}{\omega}\right)^{2} \tau_{c} \frac{\tau_{c} / \tau_{B}}{1+\left(\tau_{c} / \tau_{B}\right)^{2}}\left[f_{0}\left\{1-f_{0}\right\}\right] .
\end{aligned}
$$




\section{B. Modified results for general case}

Using Eqs. (31)-(34), we have first plotted $\eta_{1,2,3,4}$ vs $T$ in Fig. 3(a) and then their normalized values $\eta_{1,2,3,4} / s$ in Fig. 3(b). An interesting point is that all components of shear viscosity in the presence of magnetic field are smaller than their isotropic values in the absence of magnetic field. In Fig. 3(b), we notice that the KSS line is crossing different curves at different temperatures. Here, we find that at fixed $\tau_{c}$ the perfect fluid nature will be developed in quark matter at a higher temperature for $B \neq 0$ with respect to its $B=0$ case. It reflects that for fixed interaction magnetic field se the system toward the KSS bound, while temperature kicks away from the bound. To highlight the fact, we have plotted $\eta_{1,2,3,4}$ vs $e B / m_{\pi}^{2}$ in Fig. 4, in which the decreasing trend of $\eta_{1,2}$ with magnetic field is clearly observed. Magnetic fielddependent Hall-type coefficients $\eta_{3,4}$ behave a little differently because of their anisotropic structure $\frac{\left(\tau_{c} / \tau_{B}\right)}{1+\left(\tau_{c} / \tau_{B}\right)^{2}}$, which increases with $B$ for $\tau_{c} / \tau_{B}<1$ but decreases with $B$ for $\tau_{c} / \tau_{B}>1$. Therefore, we get increasing $\eta_{3,4}(B)$ for $\tau_{c}=$ $1 \mathrm{fm}$ and decreasing $\eta_{3,4}(B)$ for $\tau_{c}=20 \mathrm{fm}$, as displayed in Figs. 4(b) and 4(d).

Another interesting point has also been shown in Fig. 4. It is regarding the merging of general anisotropic shear viscosity components with their strong field limit estimation. At the strong field limit, $\eta_{2}=4 \eta_{1}$ and $\eta_{4}=2 \eta_{3}$, the expressions of which are given in Eqs. (21) and (22). These strong field limit estimations of $\eta_{2}=4 \eta_{1}$ and $\eta_{4}=2 \eta_{3}$ curves are plotted by the dotted lines in Figs. 4(a) and 4(b), respectively, for $\tau_{c}=1 \mathrm{fm}$. On the other hand, general anisotropic components of shear viscosity can be obtained from Eqs. (31)-(34), and we plotted $4 \eta_{1}$ (red dash line), $\eta_{2}$ (blue solid line), $2 \eta_{3}$ (green dash line), and $\eta_{4}$ (orange solid line) in Figs. 4(a) and 4(b) for $\tau_{c}=1 \mathrm{fm}$. We notice that these curves are not merging in any point of the $B$ axis up to

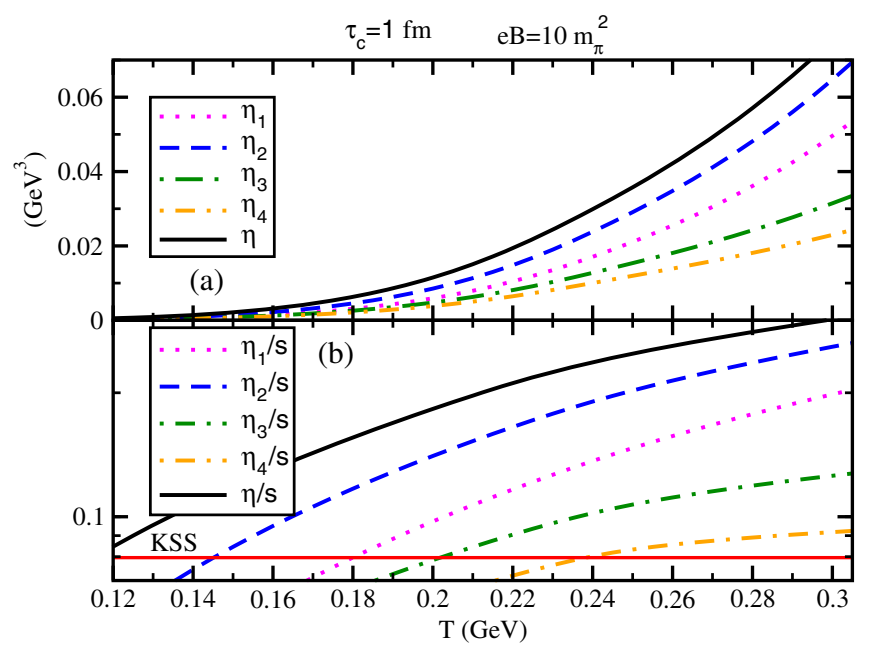

FIG. 3. (a) $T$ dependence of $\eta$ at $e B=0$ (solid line) and $\eta_{1,2,3,4}$ (dotted, dash, dash-dotted, and dash-double-dotted lines) at $e B=$ $10 m_{\pi}^{2}$. (b) Corresponding viscosity-to-entropy density ratios.
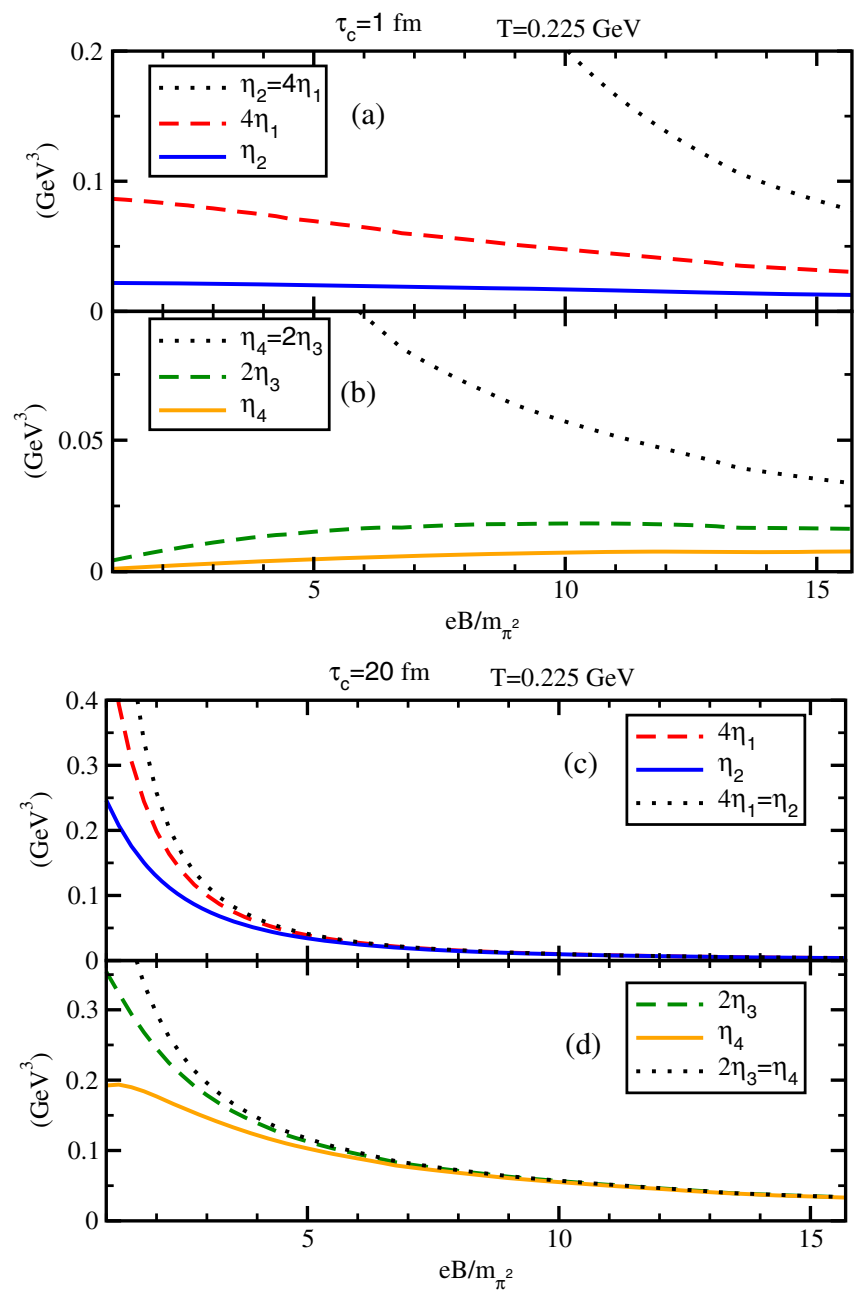

FIG. 4. For $\tau_{c}=1 \mathrm{fm}$, (a) $4 \eta_{1}, \eta_{2}$ from Eqs. (31) and (32), respectively, and its strong field limit (where $4 \eta_{1}=\eta_{2}$ ) from Eq. (21). Similarly, for $\tau_{c}=1 \mathrm{fm}$, (b) $2 \eta_{3}, \eta_{4}$ from Eqs. (33) and (34), respectively, and its strong field limit (where $2 \eta_{3}=\eta_{4}$ ) from Eq. (22). (c) and (d) are the same as (a) and (b) for $\tau_{c}=20 \mathrm{fm}$.

$e B=15 m_{\pi}^{2}$, but when we use $\tau_{c}=20 \mathrm{fm}$ in Figs. 4(c) and 4(d), they are merging after $e B=6 m_{\pi}^{2}$. So, the results say that strong field limit might be a good approximation for $\tau_{c}>10 \mathrm{fm}$.However, for RHIC or LHC matter, whose $\tau_{c}<10 \mathrm{fm}$, strong field limit expressions might not be considered a good approximated estimation. This picture will be more clear in Fig. 5, which exposes the $\tau_{c}$ dependence of $\eta_{2} / s$ from Eq. (32) (solid line) and Eq. (21) (dotted line) at $e B=10 m_{\pi}^{2}, T=0.150 \mathrm{GeV}$ in the range of (a) $\tau_{c}<10 \mathrm{fm}$ and (b) $\tau_{c}>10 \mathrm{fm}$. Here, we find how general $\eta_{2} / s$ is merging with its strong field limit curves in the second zone $\left(\tau_{c}>10 \mathrm{fm}\right)$, while they are quite far in the first zone $\left(\tau_{c}<10 \mathrm{fm}\right)$. The same qualitative pattern is also noticed for $T=0.300 \mathrm{GeV}$ in Figs. 5(c) and (d). From the crossing of the KSS line in Fig. 5, we can say that nearly perfect fluid nature can be obtained for two different values of $\tau_{c}$ within the 


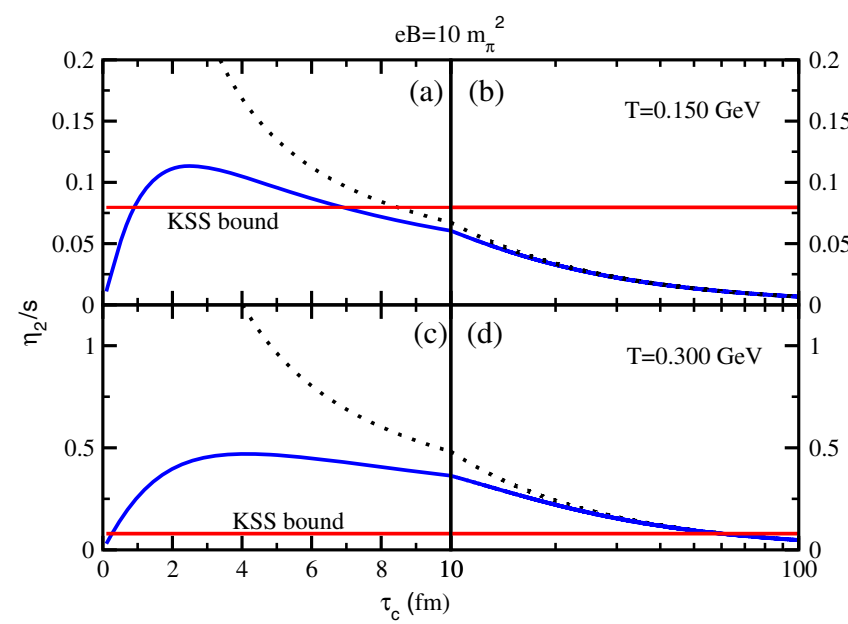

FIG. 5. $\tau_{c}$ dependence of $\eta_{2} / s$ from Eq. (32) (solid line) and Eq. (21) (dotted line) at $e B=10 m_{\pi}^{2}, T=0.150 \mathrm{GeV}$ in the range of (a) $\tau_{c}<10 \mathrm{fm}$ and (b) $\tau_{c}>10 \mathrm{fm}$ and at $T=0.300 \mathrm{GeV}$ in the range of (c) $\tau_{c}<10 \mathrm{fm}$ and (d) $\tau_{c}>10 \mathrm{fm}$. The straight horizontal red line denotes the KSS bound.

$\tau_{c}<10 \mathrm{fm}$ zone at $e B=10 m_{\pi}^{2}, T=0.150 \mathrm{GeV}$. We can denote them as $\tau_{c}^{\mp}$. The values of $\tau_{c}^{-}$are below $1 \mathrm{fm}$, and, such a value might not be reached in the RHIC or LHC matter. So, $\tau_{c}^{+}$is a more phenomenological point. We notice that $\tau_{c}^{+}$at $T=0.300 \mathrm{GeVremain}$ within the zone of $\tau_{c}>10 \mathrm{fm}$. Hence, alternatively, we can say that to build nearly perfect fluid nature in higher-temperature quark matter we need a higher magnetic field if we want it for the $\tau_{c}<10 \mathrm{fm}$ zone. It is quite possible for the RHIC or LHC matter, having the $\tau_{c}<10 \mathrm{fm}$ zone, in which a high magnetic field decays with time and temperature. This means that as we approach a higher temperature that matter can face higher magnetic field in experiments. So, there might be a compensating role of temperature and magnetic field to build nearly perfect fluid nature in the RHIC or LHC matter.

\section{SUMMARY}

We have studied the shear viscosity of quark matter in a uniform magnetic field background, where the medium loses its isotropic property. Because of this anisotropic nature, one can get more than one component of shear viscosity, denoted by $\eta_{1}, \eta_{2}, \eta_{3}$, and $\eta_{4}$, which are ultimately reduced to two main components in the strong field limit through relations $4 \eta_{1}=\eta_{2}$ and $2 \eta_{3}=\eta_{4}$. We know that isotropic shear viscosity $\eta$ in the absence of magnetic field is mainly governed by two parts: the phase space and the relaxation time. Here, also, $\eta_{2}$ and $\eta_{4}$ can be cast into the similar structure with phase space and relaxation time parts. The relaxation time of $\eta_{4}$ is inversely proportional to synchrotron frequency $\omega_{B}$, and the relaxation time of $\eta_{2}$ is $\frac{\Gamma_{c}}{\left(\omega_{B}\right)^{2}}$ in strong field limit, where the collisional thermal width $\Gamma_{c}$ of medium constituents will be much smaller that its synchrotron frequency, i.e., $\Gamma_{c} \ll \omega_{B}$. However, a large value of $\Gamma_{c}$ is expected for strongly coupled RHIC or LHC matter. To describe that zone, we need a general structure of $\eta_{1,2,3,4}$, which does not follow the relations $4 \eta_{1}=\eta_{2}$ and $2 \eta_{3}=\eta_{4}$ below the strong field domain.

We have used the formalism of the NJL model in the presence of magnetic field to describe the magnetothermodynamics of quark matter, and we get a temperature- and magnetic field-dependent quark mass, which will be entered to the phase space factors of $\eta_{1,2,3,4}$. In strong field limits, all components decrease with $B$, but in the weak field case, the Hall-type viscosities $\eta_{3,4}$ increase with $B$.

Along with the constant value $\tau_{c}$, we have also calculated the $T$ dependence of $\tau_{c}$ from a simple contact diagram of $2 \rightarrow 2$ scattering processes, coming from the interaction Lagrangian density of the NJL model. Replacing the $T$-dependent quark mass $M_{Q}(T)$ by $T$, the $e B$-dependent quark mass $M_{Q}(T, e B)$, we have extended the expression of relaxation time from $\tau_{c}(T)$ to $\tau_{c}(T, e B)$. Scattering probability $\left(\Gamma_{c}\right)$ proportionally increases with the density of the medium, which increases with $T$ due to statistical reasons and decreases with $e B$ due to mass enhancement. Hence, relaxation time $\tau_{c}(T, e B)$ decreases with $T$ and increases with $e B$. In the absence of magnetic field, the shear viscosity-to-entropy density ratio decreases with $T$ as it is proportional to relaxation time, but it increases with $T$ in the strong field picture as it is inversely proportional to the relaxation time. So, when we shift from zero to finite magnetic field picture, $T$ dependence of viscosity to entropy density ratio transforms from its decreasing to increasing trends.

In the present work, we have calculated $\tau_{c}(T, e B)$ from the simplest contact diagram $2 \rightarrow 2$ scattering processes, which provide a large $\tau_{c}$, where $e B=10 m_{\pi}^{2}$ can safely be considered a strong field case. However, to describe RHIC or LHC matter with small $\tau_{c}$, we have to consider the general structure of $\eta_{1,2,3,4}$ and a better interaction picture, which can map strongly coupled matter. In future, we are planning to build a better interaction picture through Feynmann diagram calculations in presence of magnetic field.

\section{ACKNOWLEDGMENTS}

S. G. acknowledges to Indian Institute of Technology Bhilai, funded by Ministry of Human Resource Development as well as an earlier D. S. Kothari fellowship, University Grants Commission, under Grant No. F.4-2/ 2006 (BSR)/PH/15-16/0060. S. G. and A. M. thank Pracheta Singha for initial constructive discussion on this work. 
[1] T. Schafer and D. Teaney, Rep. Prog. Phys. 72, 126001 (2009).

[2] K. Tuchin, Adv. High Energy Phys. 2013, 490495 (2013).

[3] V. Skokov, A. Y. Illarionov, and V. Toneev, Int. J. Mod. Phys. A 24, 5925 (2009).

[4] V. Voronyuk, V. D. Toneev, W. Cassing, E. L. Bratkovskaya, V. P. Konchakovski, and S. A. Voloshin, Phys. Rev. C 83, 054911 (2011).

[5] A. Bzdak and V. Skokov, Phys. Lett. B 710, 171 (2012).

[6] W. T. Deng and X. G. Huang, Phys. Rev. C 85, 044907 (2012).

[7] W. T. Deng and X. G. Huang, Phys. Lett. B 742, 296 (2015).

[8] J. O. Andersen, W. R. Naylor, and A. Tranberg, Rev. Mod. Phys. 88, 025001 (2016).

[9] V. A. Miransky and I. A. Shovkovy, Phys. Rep. 576, 1 (2015).

[10] R. Gatto and M. Ruggieri, Phys. Rev. D 83, 034016 (2011).

[11] R. Gatto and M. Ruggieri, Lect. Notes Phys. 871, 87 (2013).

[12] J. K. Boomsma and D. Boer, Phys. Rev. D 81, 074005 (2010).

[13] B. Chatterjee, H. Mishra, and A. Mishra, Phys. Rev. D 84, 014016 (2011).

[14] B. Chatterjee, H. Mishra, and A. Mishra, Phys. Rev. D 91, 034031 (2015).

[15] G. S. Bali, F. Bruckmann, G. Endrodi, Z. Fodor, S. D. Katz, S. Krieg, A. Schafer, and K. K. Szabo, J. High Energy Phys. 02 (2012) 044.

[16] V. G. Bornyakov, P. V. Buividovich, N. Cundy, O. A. Kochetkov, and A. Schafer, Phys. Rev. D 90, 034501 (2014).

[17] A. Ayala, M. Loewe, A. Z. Mizher, and R. Zamora, Phys. Rev. D 90, 036001 (2014).

[18] A. Ayala, M. Loewe, and R. Zamora, Phys. Rev. D 91, 016002 (2015).

[19] R. L. S. Farias, K. P. Gomes, G. I. Krein, and M. B. Pinto, Phys. Rev. C 90, 025203 (2014).

[20] R. L. S. Farias, V. S. Timoteo, S. S. Avancini, M. B. Pinto, and G. Krein, Eur. Phys. J. A 53, 101 (2017).

[21] S. Li and H-U. Yee, Phys. Rev. D 97, 056024 (2018).

[22] S. Nam and C-W. Kao, Phys. Rev. D 87, 114003 (2013).

[23] M. G. Alford, H. Nishimura, and A. Sedrakian, Phys. Rev. C 90, 055205 (2014).

[24] A. N. Tawfik, A. M. Diab, and M. T. Hussein, Int. J. Adv. Res. Phys. Sci. 3, 4 (2016).

[25] K. Tuchin, J. Phys. G 39, 025010 (2012).

[26] K. Hattori, X. G. Huang, D. H. Rischke, and D. Satow, Phys. Rev. D 96, 094009 (2017).

[27] X-G. Huang, M. Huang, D. H. Rischke, and A. Sedrakian, Phys. Rev. D 81, 045015 (2010).
[28] X. G. Huang, A. Sedrakian, and D. H. Rischke, Ann. Phys. (Amsterdam) 326, 3075 (2011).

[29] N. O. Agasian, Phys. At. Nucl. 76, 1382 (2013).

[30] N. O. Agasian, JETP Lett. 95, 171 (2012).

[31] S. i. Nam, Phys. Rev. D 86, 033014 (2012).

[32] K. Hattori and D. Satow, Phys. Rev. D 94, 114032 (2016).

[33] K. Hattori, S. Li, D. Satow, and H.-U. Yee, Phys. Rev. D 95, 076008 (2017).

[34] A. Harutyunyan and A. Sedrakian, Phys. Rev. C 94, 025805 (2016).

[35] B. O. Kerbikov and M. A. Andreichikov, Phys. Rev. D 91, 074010 (2015).

[36] B. Feng, Phys. Rev. D 96, 036009 (2017).

[37] P. V. Buividovich, M. N. Chernodub, D. E. Kharzeev, T. Kalaydzhyan, E. V. Luschevskaya, and M. I. Polikarpov, Phys. Rev. Lett. 105, 132001 (2010).

[38] K. Fukushima and Y. Hidaka, arXiv:1711.1472.

[39] V. Roy, S. Pu, L. Rezzolla, and D. Rischke, Phys. Lett. B 750, 45 (2015).

[40] S. Pu, V. Roy, L. Rezzolla, and D. H. Rischke, Phys. Rev. D 93, 074022 (2016).

[41] S. K. Das, S. Plumari, S. Chatterjee, J. Alam, F. Scardina, and V. Greco, Phys. Lett. B 768, 260 (2017).

[42] P. Mohanty, A. Dash, and V. Roy, Eur. Phys. J. A 55, 35 (2019).

[43] R. Critelli, S. I. Finazzo, M. Zaniboni, and J. Noronha, Phys. Rev. D 90, 066006 (2014); S. I. Finazzo, R. Critelli, R. Rougemont, and J. Noronha, Phys. Rev. D 94, 054020 (2016).

[44] S. Jain, R. Samanta, and S. P. Trivedi, J. High Energy Phys. 10 (2015) 028.

[45] E. M. Lifshitz and L.P. Pitaevskii, Physical Kinetics (Pergamon, New York, 1987).

[46] M. Frank, M. Buballa, and M. Oertel, Phys. Lett. B 562, 221 (2003).

[47] S. Gavin, Nucl. Phys. A435, 826 (1985).

[48] P. Chakraborty and J. I. Kapusta, Phys. Rev. C 83, 014906 (2011).

[49] A. Abhishek, H. Mishra, and S. Ghosh, Phys. Rev. D 97, 014005 (2018).

[50] P. Singha, A. Abhishek, G. Kadam, S. Ghosh, and H. Mishra, J. Phys. G 46, 015201 (2019).

[51] P. Deb, G. P. Kadam, and H. Mishra, Phys. Rev. D 94, 094002 (2016).

[52] S. Ghosh, T. C. Peixoto, V. Roy, F. E. Serna, and G. Krein, Phys. Rev. C 93, 045205 (2016).

[53] S. Ghosh, F. E. Serna, A. Abhishek, G. Krein, and H. Mishra, Phys. Rev. D 99, 014004 (2019). 